\title{
Erratum to: Nucleation and Growth of Graphite in Eutectic Spheroidal Cast Iron: Modeling and Testing
}

\author{
FERNANDO D. CARAZO, PATRICIA M. DARDATI, DIEGO J. CELENTANO, \\ and LUIS A. GODOY
}

DOI: $10.1007 / \mathrm{s} 11661-016-3632-2$

(C) The Minerals, Metals \& Materials Society and ASM International 2016

\section{Erratum to: METALLURGICAL AND MATERIALS \\ TRANSACTIONS A, Volume 47A, June \\ 2016, pp. 2625-2641 \\ DOI 10.1007/s11661-016-3430-x}

THE following are corrections to the original article:

In Appendix A, temperature formulas

$$
\begin{aligned}
& \mathrm{T}_{\mathrm{a}_{\mathrm{T}}}^{\alpha}=-\left(\sqrt{1.10 \times 10^{11}+2.74 \times 10^{35}\left(\mathrm{C}_{\mathrm{Si}}\right)^{2}-3.25 \times 10^{35} \mathrm{C}_{\mathrm{Si}}+1.46 \times 10^{34}}+1.74 \times 10^{23} \mathrm{C}_{\mathrm{Si}}-1.02 \times 10^{23}\right) / 8.53 \times 10^{19} \\
& \mathrm{~T}_{\mathrm{A}_{1}}^{\alpha}=-\left(\sqrt{3.47 \times 10^{11}+1.60 \times 10^{22}\left(\mathrm{C}_{\mathrm{Si}}\right)^{2}-2.19 \times 10^{21} \mathrm{C}_{\mathrm{Si}}+5.85 \times 10^{19}}+7.4 \times 10^{16} \mathrm{C}_{\mathrm{Si}}-9.85 \times 10^{15}\right) / 7.69 \times 10^{12} \\
& \mathrm{~T}_{\mathrm{a}_{\mathrm{T}}}^{\mathrm{P}}=-\left(\sqrt{2.16 \times 10^{10}+5.07 \times 10^{20}\left(\mathrm{C}_{\mathrm{Si}}\right)^{2}-3.94 \times 10^{20} \mathrm{C}_{\mathrm{Si}}+5.23 \times 10^{19}}+3.08 \times 10^{15} \mathrm{C}_{\mathrm{Si}}-2.82 \times 10^{15}\right) / 2.32 \times 10^{12} \\
& \mathrm{~T}_{\mathrm{A}_{1}}^{\mathrm{P}}=-\left(\sqrt{4.05 \times 10^{11}+6.72 \times 10^{21}\left(\mathrm{C}_{\mathrm{Si}}\right)^{2}-7.3 \times 10^{20} \mathrm{C}_{\mathrm{Si}}+7.5 \times 10^{19}}+5.46 \times 10^{16} \mathrm{C}_{\mathrm{Si}}+1.16 \times 10^{16}\right) / 2.4 \times 10^{13}
\end{aligned}
$$

\footnotetext{
FERNANDO D. CARAZO, Professor and Researcher, is with the Instituto de Mecánica Aplicada, Universidad Nacional de San Juan, Av. Libertador Gral. San Martín 1109 (Oeste), C.P. J5400ARL, San Juan, Argentina, and also with the CONICET, C1425FQB Godoy Cruz 2290, CABA Argentina. Contact e-mail: fcarazo@unsj.edu.ar PATRICIA M. DARDATI, Professor, is with the GIDMA and Departamento de Ingeniería Mecánica, Universidad Tecnológica Nacional Facultad Regional Córdoba, Maestro M. López esq. Cruz Roja Argentina s/n, Ciudad Universitaria, Córdoba, Argentina. DIEGO J. CELENTANO, Professor, is with the Departamento de Ingeniería Mecánica y Metalúrgica, Research Center for Nanotechnology and Advanced Materials (CIEN-UC), Pontificia Universidad Católica de Chile, Av. Vicuña Mackenna 4860, Macul, Santiago, Chile. LUIS A. GODOY, Professor and Researcher, is with the Institute for Advanced Studies in Engineering and Technology, FCEFyN, National University of Cordoba and CONICET, Av. Vélez Sarsfield 1601, Córdoba, Argentina, and also with the CONICET.

The online version of the original article can be found under doi: 10.1007/s11661-016-3430-x.

Article published online July 25, 2016
} 
should be replaced by

$\mathrm{T}_{\mathrm{a}_{\mathrm{T}}}^{\alpha}=-\left(\sqrt{1.10 \times 10^{11}} \sqrt{2.74 \times 10^{35}\left(\mathrm{C}_{\mathrm{Si}}\right)^{2}-3.25 \times 10^{35} \mathrm{C}_{\mathrm{Si}}+1.46 \times 10^{34}}+1.74 \times 10^{23} \mathrm{C}_{\mathrm{Si}}-1.02 \times 10^{23}\right) / 8.53 \times 10^{19}$ $\mathrm{T}_{\mathrm{A}_{1}}^{\alpha}=-\left(\sqrt{3.47 \times 10^{11}} \sqrt{1.60 \times 10^{22}\left(\mathrm{C}_{\mathrm{Si}}\right)^{2}-2.19 \times 10^{21} \mathrm{C}_{\mathrm{Si}}+5.85 \times 10^{19}}+7.4 \times 10^{16} \mathrm{C}_{\mathrm{Si}}-9.85 \times 10^{15}\right) / 7.69 \times 10^{12}$ $\mathrm{T}_{\mathrm{a}_{\mathrm{T}}}^{\mathrm{P}}=-\left(\sqrt{2.16 \times 10^{10}} \sqrt{5.07 \times 10^{20}\left(\mathrm{C}_{\mathrm{Si}}\right)^{2}-3.94 \times 10^{20} \mathrm{C}_{\mathrm{Si}}+5.23 \times 10^{19}}+3.08 \times 10^{15} \mathrm{C}_{\mathrm{Si}}-2.82 \times 10^{15}\right) / 2.32 \times 10^{12}$ $\mathrm{T}_{\mathrm{A}_{1}}^{\mathrm{P}}=\left(\sqrt{4.05 \times 10^{11}} \sqrt{6.72 \times 10^{21}\left(\mathrm{C}_{\mathrm{Si}}\right)^{2}-7.3 \times 10^{20} \mathrm{C}_{\mathrm{Si}}+7.5 \times 10^{19}}+5.46 \times 10^{16} \mathrm{C}_{\mathrm{Si}}+1.16 \times 10^{16}\right) / 2.4 \times 10^{13}$

In Appendix B, Table B-IV, diffusion coefficient of carbon in the liquid $\left(\mathrm{D}_{\mathrm{C}}^{1}=5 \times 10^{10}\right)$ is corrected as $\mathrm{D}_{\mathrm{C}}^{1}=5 \times 10^{-10}$. 